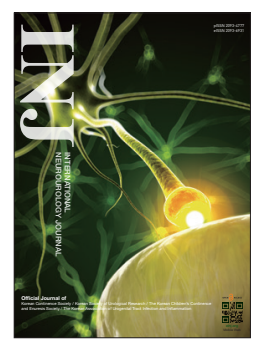

\title{
Time-Dependent Changes of Urethral Function in Diabetes Mellitus: A Review
}

\author{
Nailong $\mathrm{Cao}^{1,2}$, Baojun Gu${ }^{1}$, Daisuke Gotoh², Naoki Yoshimura² \\ ${ }^{1}$ Department of Urology, Shanghai Jiao Tong University Affiliated Sixth People’s Hospital, Shanghai, China \\ ${ }^{2}$ Department of Urology, University of Pittsburgh School of Medicine, Pittsburgh, PA, USA
}

\begin{abstract}
This article reviewed the current knowledge on time-course manifestation of diabetic urethral dysfunction (DUD), and explored an early intervention target to prevent the contribution of DUD to the progression of diabetes-induced impairment of the lower urinary tract (LUT). In the literature search through PubMed, key words used included "diabetes mellitus," "diabetic urethral dysfunction," and "diabetic urethropathy." Polyuria and hyperglycemia induced by diabetes mellitus (DM) can cause the time-dependent changes in functional and morphological manifestations of DUD. In the early stage, it promotes urethral dysfunction characterized by increased urethral pressure during micturition. However, the detrusor muscle of the bladder tries to compensate for inducing complete voiding by increasing the duration and amplitude of bladder contractions. As the disease progresses, it can induce an impairment of coordinated micturition due to dyssynergic activity of external urethra sphincter, leading to detrusor-sphincter dyssynergia. The impairment of relaxation mechanisms of urethral smooth muscles (USMs) may additionally be attributable to decreased responsiveness to nitric oxide, as well as increased USM responsiveness to $a_{1}$-adrenergic receptor stimulation. In the late stage, diabetic neuropathy may play an important role in inducing LUT dysfunction, showing that the decompensation of the bladder and urethra, which can cause the decrease of voiding efficiency and the reduced thickness of the urothelium and the atrophy of striated muscle bundles, possibly leading to the vicious cycle of the LUT dysfunction. Further studies to increase our understandings of the functional and molecular mechanisms of DUD are warranted to explore potential targets for therapeutic intervention of DM-induced LUT dysfunction.
\end{abstract}

Keywords: Diabetes mellitus; Lower urinary tract symptoms; Urethral dysfunction

- Grant/Fund Support: This work was supported by National Natural Science foundation of China (Grant No. 81870521, 81370862) and Natural Science Foundation of Shanghai (Grant No. 18ZR1428900). Fund from the Doctorate Innovation Fund of Shanghai Jiao Tong University School of Medicine (Grant No. BXJ201840) and China Scholarship Council.

- Conflict of Interest: No potential conflict of interest relevant to this article was reported.

\section{INTRODUCTION}

Diabetes mellitus (DM) is characterized by chronic hyperglycemia due to insulin deficiency in the case of type 1 diabetes or insulin resistance in the case of type 2 diabetes. It is projected that there are more than 150 million in the world [1]. As many as $80 \%$ with noninsulin dependent DM have cystopathy, which is characterized by impaired sensations of bladder fullness, in-
Corresponding author: Baojun $\mathrm{Gu}$ (D) https://orcid.org/0000-0001-5788-452X Department of Urology, Shanghai Jiao Tong University Affiliated Sixth People's Hospital, 600 Yishan Road, Shanghai 200233, China

E-mail: gubaojun@yahoo.com / Tel: +86-21-24058382 / Fax: +86-21-64083783

Co-Corresponding author: Naoki Yoshimura (iD http://orcid.org/0000-0001-8070-1664 Department of Urology, University of Pittsburgh School of Medicine, Suite 700,

Kaufmann Medical Bldg 3471 Fifth Ave, Pittsburgh, PA, USA

E-mail: nyos@pitt.edu / Tel: +1-412-692-4137 / Fax: +1-412-692-4380

Submitted: February 20, 2019 / Accepted after revision: March 4, 2019 (c) (1) $\$$ This is an Open Access article distributed under the terms of the Creative Commons Attribution Non-Commercial License (http://creativecommons.org/licenses/by-nc/4.0/) which permits unrestricted non-commercial use, distribution, and reproduction in any medium, provided the original work is properly cited. 
creased bladder capacity, decreased bladder contractility, and elevated postvoid residual urine volume [2,3]. Moreover, functional and morphological manifestations of diabetic bladder dysfunction (DBD) in studies of streptozotocin (STZ)-induced DM animal model are time-dependent [4]. Bladder hypertrophy and remodeling, increased contractility and associated neurogenic changes occur soon after the onset of DM $[5,6]$, while decreased peak voiding pressure in the cystometric measure develops only at a later stage of DM [4,7]. However, compared with this well documented DBD, little is known about the effects of DM on urethral function.

Previous animal studies have demonstrated that DM could also damage the innervation of the urethra and directly impairs urethral striated and smooth muscles $[8,9]$. The resulting diabetic urethral dysfunction (DUD) could degrade voiding function by impairing outlet function and urethra-to-bladder reflexes [10]. DM induce an impairment of coordinated micturition consisting of external urethra sphincter (EUS) phasic activity and urethra smooth muscle (USM) relaxation mechanisms, resulting in detrusor-sphincter dyssynergia (DSD), which is an involuntary contraction of the EUS during detrusor contraction $[11,12]$. However, the time-dependent functional and morphological changes in DUD have not been well characterized, and the alterations of neurotransmitter systems and urethra-to-bladder reflexes have not been well clarified.

Our main purpose is therefore to review the previous literatures regarding time-dependent changes of urethral function in DM and explore an early intervention target to prevent the urethral dysfunction to the progression of DM-induced damage to the lower urinary tract (LUT).

\section{EPIDEMIOLOGICAL STUDY}

In 2015, the International Diabetes Federation (IDF) estimated that approximately 415 million adults aged $20-79$ years (about $8.8 \%$ ) had DM worldwide and that the condition accounted for $12 \%$ of global healthcare expenditure. The IDF predicts that, if current trends continue, by 2040 one in 10 adults will have DM [10]. More than 29 million people in the United States (US) have DM and 86 million of US adults have prediabetic conditions including metabolic syndrome, according to the Centers for Disease Control and Prevention National Diabetes Fact Sheet. During recent decades, rapid economic development and urbanization have also increased the prevalence of DM in Asia. For example, China has the highest diabetes prevalence worldwide, with more than 98 million people being affected. The prevalence of DM has increased significantly in recent decades and is now reaching epidemic proportions in China $[13,14]$. Furthermore, it is worth mentioning that the number is projected to rise to nearly 143 million by 2035 [15]. Although different sampling methods, screening procedures, and diagnostic criteria were used, these data document a rapid increase in DM.

Macrovascular and microvascular complications of DM, including cardiovascular diseases, retinopathy, nephropathy, and neuropathy, have been well investigated [16,17]. DM also contributes to the earlier onset and increased severity of urologic conditions, leading to debilitating urologic complications, including LUT dysfunction [12,18]. LUT complications are found in more than $80 \%$ of individuals diagnosed with $\mathrm{DM}$, a higher rate than that of widely recognized complications such as neuropathy and nephropathy, which affect less than $60 \%$ and $50 \%$, respectively [19] Although the most common, bothersome LUT complication of DM is DBD, DUD can also cause LUT dysfunction and the effects of DUD should be given attention.

\section{PRECLINICAL STUDY}

\section{Functional changes of the urethra in DUD}

Studies of the effects of DM on LUT function have largely focused on the bladder. As noted, the bladder is only one important component of LUT. The urethra, which serves as the bladder outlet, must remain closed during storage to maintain continence and open to provide a low resistance outlet during voiding. Thus, any dysfunction of urethral closing or opening adversely affects continence or voiding function, respectively, and it must be considered when assessing the effects of DM on LUT function.

STZ-treated DM rats have been extensively used as a rodent model of the voiding dysfunction encountered in DM patients. In this model, there are time-dependent morphological and functional changes in myogenic and neurogenic components of the bladder and urethra that affect both voiding and storage phases $[4,20,21]$. DM may initially promote urethral dysfunction characterized by increased urethral pressure during micturition. Urethral pressure showed a significant increase in 5-week STZ-induced DM rats, and this may be attributed to the impairment of the USM relaxation mechanism because administration of $\mathrm{L}$-arginine, the substrate of nitric oxide (NO), can significantly partially restore the urethral relaxation and im- 
prove voiding efficiency by [8].

It is noted that elevation of urethral pressure during the micturition reflex can increase the bladder outlet resistance, and the bladder detrusor tries to compensate to maintain efficient voiding by increasing the duration of bladder contraction. The peak contraction amplitude also increased in cystometrogram measurements, and EUS-electromyogram (EMG) recordings showed increased frequency of EUS-EMG bursting discharge during voiding in 6-week DM rats [22]. DSD also occurred in approximately $30 \%$ of the 5 and 10 weeks STZ-induced DM rats, which suggested that DM could lead to EUS dysfunction, decreased USM relaxation with reduced NO responsiveness, and increased urethral smooth muscle responsiveness to $\alpha_{1}$ adrenergic stimulation [12]. Interestingly, the insensitivity to NO responsiveness and hypersensitivity to $\alpha_{1}$-adrenergic agonists were more common in 10-week DM rats, suggesting that urethropathy is evident in both short-term and long-term STZ induced DM rats [12].

Few studies have investigated longer-term alterations in the urethra secondary to DM. In a previous study, DM changed the EUS-EMG pattern in a different manner at 6 and 20 weeks after STZ induction [22]. The differences in EUS-EMG patterns between 6- and 20-week diabetic rats might be caused by different mechanisms. Early changes in micturition function in DM may be attributable to functional adaptation to an increase in blad- der work and overdistention arising from hyperglycemia-induced diuresis $[5,23]$. However, diabetic neuropathy may play an important role in inducing LUT changes in late stages of DM [3]. Furthermore, the decreased voiding efficiency can be attributable to chronic hyperglycemia, which induces a decompensated, hypertrophied detrusor muscle. DM-induced neuropathy may also contribute to the decompensated bladder whose contraction is inadequate to expel the increased bladder capacity since it has been reported that DM cystopathy is associated with polyneuropathy affecting autonomic nerve fiber [3]. Taken together, potential mechanisms inducing early- and latephases of DUD are summarized in Fig. 1, mainly based on previous preclinical study data.

\section{Morphological changes of the urethra in DUD}

To better understand the mechanistic nature of DM urethropathy, we must understand the function of the normal urethra [10,24-26]. In this regard, the urethra is composed of 3 muscle layers, including the proximal USM-Longitudinal (USM-L), which is the innermost and extends into the bladder base, the USM-Circumferential (USM-C), which has its greatest thickness at the mid urethra, and the striated EUS, which is also circular in orientation and surrounds the distal urethra. While EUS relaxation is passive due to cessation of its excitation, the opening of the urethral smooth muscle conduit is not a passive

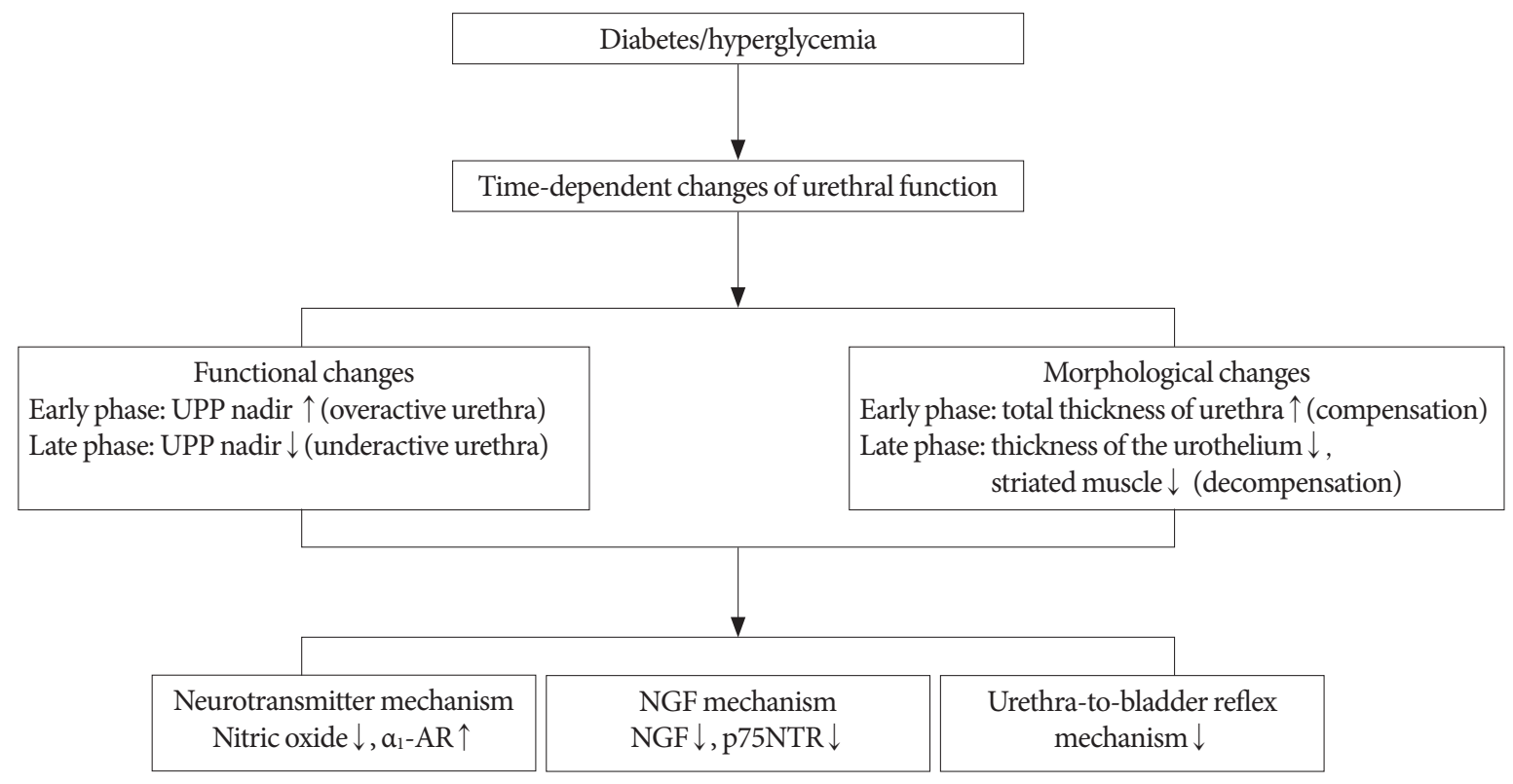

Fig. 1. Potential mechanisms inducing the time-dependent changes of urethral function in diabetes mellitus (DM). UPP, urethral perfusion pressure; $\alpha_{1}-A R, \alpha_{1}$-adrenergic receptor; NGF, nerve growth factor; p75NTR, p75 neurotrophin receptor. 
event but rather requires active USM-L contraction (mediated by acetylcholine $[\mathrm{Ach}]$ and/or prostaglandin E2 $[27,28])$ and active USM-C relaxation (mediated by NO [29]). Urethral closure clearly depends on active USM-C contraction (mediated by norepinephrine [NE] through $\alpha$-adrenergic receptors) and may or may not rely on active mechanisms in terms of USM-L relaxation.

Previous studies reported that the morphology of the urethra can also be altered in DM. For example, the urethras from 6 weeks and 20 weeks in alloxan-induced diabetic rats had greater values of total thickness than did the control rats whereas the urethra from 6- and 20-week DM rats had reduced thickness of the urothelium of the urethra [30]. In 6- and 20-week STZ-induced DM rats, the morphological images revealed that the striated muscle bundles in 20-week diabetic rats were atrophied compared with those of controls although no obvious changes were observed in the thickness of EUS of 6-week diabetic rats compared with controls (Fig. 1) [22].

Few studies have investigated the longer-term morphological alterations in the urethra secondary to DM. A higher total thickness and muscle layer thickness and higher connective tissue and collagen content were observed in the urethras in 44week alloxan-induced DM rats. However, no changes in the collagen type III/I ratio were found in the urethra between DM rats and age-matched normal rats [31]. Notably, some researchers have reported that either long-term mild hyperglycemia or short-term severe hyperglycemia have detrimental impacts on muscle health. They are both involved in the failure to maintain healthy skeletal muscle that may contribute to the development of pelvic floor dysfunctions via different pathways [32].

\section{The changes of neurotransmitter and growth factor mechanisms in DUD}

The 2 main functions of the LUT, and the storage and periodic expulsion of urine, are dependent on the coordinated activity of the urinary bladder and urethra $[33,34]$. Voiding is accomplished by the activation of the parasympathetic efferent neurons which release ACh to contract the bladder smooth muscle and NO to relax the USM. Simultaneously, the excitatory input to EUS from spinal motor neurons is inhibited to allow urine flow. Storage of urine involves tonic activation of the sympathetic system, which releases NE. NE acts on beta adrenergic receptors ( $\beta$-ARs) to relax the bladder smooth muscle and on $\alpha_{1}$-adrenergic receptors ( $\alpha_{1}$-ARs) to contract the USM. In addition, the excitatory motor input to the EUS is turned on to maintain continence, especially when stress is applied to the bladder.

Nerve growth factor (NGF) also plays an important role in the growth, survival and development of sympathetic and sensory peripheral neurons. Moreover, rats with STZ-induced DM showed a significant, time-dependent decrease in NGF levels in the bladder and L6 to S1 dorsal root ganglia [35]. Therefore, we reviewed the time-dependent changes of the neurotransmitters and its corresponding receptors in DUD.

\section{Nitric oxide}

NO is synthesized by a family of enzymes known as nitric oxide synthase (NOS). Three distinct isoforms of NOS, namely endothelial NOS (eNOS), neuronal NOS (nNOS), and inducible NOS (iNOS), have been described, based on the cells in which they were first identified, isolated, purified, and cloned [36]. nNOS is specifically expressed in neurons of the nervous system, which cause the generation of nNOS-derived NO [37]. nNOS-derived NO exhibits modulatory effects on parasympathetic nerves, which mainly act as a crucial effect on urethral relaxation during micturition reflex [38].

NO is thought to play a role in peripheral neurotransmission, and it is believed to be an important neurotransmitter in nonadrenergic, noncholinergic (NANC) nerves of the peripheral nervous system. Evidence that $\mathrm{NO}$ is a neurotransmitter in NANC nerve-mediated smooth muscle relaxation comes from studies of the rat anococcygeus muscle in which the NOS inhibitor L-NMMA abolishes nerve-mediated relaxation; this response is reversed by L-arginine [39]. Abnormalities in the NOmediating mechanisms in the cavernosal, gastric, duodenum, and anococcygeus smooth muscle have been identified in DM. Since NO plays an important role in modulating bladder neck and USM tone, it is clearly of interest to investigate its role in the pathogenesis of the urethral dysfunction associated with DM.

NO can diffuse into the smooth musculature of the urethra, in which it stimulates the synthesis of cyclic guanosine monophosphate (cGMP) and finally induces smooth muscle cell relaxation [40]. L-arginine therapy, which could augment urethral smooth muscle relaxation by increasing NO production, may be useful for partially restoring the urethral relaxation mechanism due to increased formation of NO mediated cyclic GMP formation in the urethra in 5-week STZ-induced diabetic rats [8]. Moreover, a recent study showed that DM induced elevation of urethral pressure and dysfunction of urethral relaxation 
in 8-week STZ-induced DM rats, and grape seed proanthocyanidin extract alleviated urethral dysfunction through modulating the NO-cGMP pathway [41]. Furthermore, DUD decreased urethral smooth muscle relaxation and NO responsiveness in 5- and 10-week STZ-induced DM rats, suggesting that DUD is time-dependent, and early intervention targeting bladder outlet resistance may be indicated for the therapy of DMinduced LUT dysfunction [12].

\section{Norepinephrine}

Sympathetic postganglionic terminals, which release NE, elicit contractions of the bladder base and USM, and it has been reported that the relaxation of the bladder body mediated mainly though $\beta_{3}$-ARs. Moreover, selective $\beta_{3}$-AR agonists effectively relaxes human detrusor muscle [42], indicating that the most important $\beta$-AR for bladder relaxation is the $\beta_{3}-\mathrm{AR}$, at least in humans. However, in urethral and prostatic smooth muscle, sympathetic excitation is mediated by an $\alpha_{1}$-AR subtype to induce contractions [43]. Furthermore, sphincter function is also modulated by the spinal noradrenergic pathways, and NE-induced increase in excitability of urethral rhabdosphincter motoneurons in the spinal cord may be partially through $\alpha_{1}$-ARdependent depolarization in rats [44].

According to a previous study, the relaxation mechanisms of urethral striated and smooth muscles during the micturition reflex were impaired in diabetic rats, but inhibition of $\alpha_{1}$-ARs could decrease the lowest urethral pressure (urethral perfusion pressure [UPP] nadir) and UPP fluctuation, suggesting that $\alpha_{1}$ ARs are a potential intervention target for urethral dysfunction in DM [9]. Moreover, DUD could increase responsiveness to $\alpha_{1}$-adrenergic agonists in both 5- and 10-week STZ-induced DM rats [12]. Thus, it is assumed that early inhibition of $\alpha_{1}$-ARs may be useful to improve DUD.

In addition, UPP nadir during urethral relaxation was significantly smaller in 8-week diabetic rats, while intravenous administration of tamsulosin, an $\alpha_{1}$-AR antagonist, significantly decreased the UPP nadir and baseline UPP. Therefore, it is assumed that the upregulation of the $\alpha_{1 A^{-}}$and $\alpha_{1 B}$-ARs in the urethras may be a contributing factor to DUD [45] although the exact mechanism of $\alpha_{1}$-AR involved in DUD need be further explored.

\section{Nerve growth factor}

NGF is a protein with tropism for effector tissues and their innervations $[46,47]$. NGF plays an important role in the growth, survival and development of sympathetic and sensory peripheral neurons $[48,49]$. In the urinary tract, NGF is produced in smooth muscle cells and urothelial cells of the bladder. In the urothelium, it can trigger changes in afferent fibers, either by reducing or increasing their excitability [50]. NGF induces the uptake of calcium ions which, besides releasing catecholamines, are important in maintaining smooth muscle tonus of the urethra [51].

Previous studies have also revealed that deficient retrograde axonal transport of NGF from target organs to sensory pathways may be involved in diabetic neuropathy $[52,53]$. It has been showed that down-regulated NGF expression in the urinary bladder of DM rats leads to decreased NGF transport to its afferent pathways, which contributes to diabetic cystopathy [54]. Rats with STZ-induced DM showed a significant time-dependent decrease in NGF levels in the bladder and L6 to S1 dorsal root ganglia that was associated with voiding dysfunction attributable to defects in A- and C-fiber bladder afferents [35]. However, urine NGF level was increased, and this may be due to inflammation and apoptosis in the bladder, which could cause the loss of NGF binding receptors and increased expulsion of NGF in bladder [55]. Thus, it is possible that increased urine NGF may affect the urethral function. Furthermore, a previous study revealed that the expression of NGF and p75 neurotrophin receptor level were significantly decreased in the urethras from the 8-week diabetic rats [45]. However, further studies are needed to explore whether a time-dependent decrease of urethral NGF levels is observed and involved in DUD.

Taken together, the above-mentioned neurotransmitters and its corresponding receptors undergo time-dependent changes to induce DUD (Fig. 1); however, the changes of other neurotransmitters or growth factors, such as ACh, brain-derived neurotrophic factor and serotonin have not been established. Further studies are needed to clarify these points.

\section{The changes of urethra-to-bladder reflexes in DUD}

Abundant evidence demonstrated that the sensory input generated in the urethra has a relevant impact on bladder activity $[34,56]$. Urethral anesthesia prevented normal bladder emptying of healthy subjects [57], which suggests that changes in urethro-vesical crosstalk can occur in DM and should be given attention. Several studies have demonstrated DM-induced damage to bladder afferent neurons, with A- and C-fiber afferent neurons being injured and the responses to chemical stimulation with capsaicin and acetic acid being reduced [35,58], 
therefore, similar damage to urethral afferent neurons would be expected.

In 10-week STZ-induced DM rats, chemical stimulation of urethral afferent neurons with capsaicin or dilute acetic acid showed that urethral afferent neuronal function was altered. Moreover, DM affected urethral afferent neurons differentially, compromising those expressing TRPV1 receptors. Low-frequency oscillations of USM are known to be neurogenically mediated and can increase EUS activity, revealing the existence of a hitherto undescribed reflex pathway: a smooth-to-striated muscle urethra-to-urethral reflex. Furthermore, in 9- and 12week STZ-induced DM rats, nitrergic relaxations and adrenergic-induced contractions in the isolated diabetic rat urethra display similar properties to controls, suggesting no dysfunction on the nitrergic or $\alpha_{1}$-AR function in the smooth muscle. This further implies that compromised urethral relaxation and increased adrenergic agonist sensitivity observed in vivo in this model may be due to the disruption of neural signaling between the urethra and the spinal cord, or within the central nervous system (CNS) [59]. Thus, DM-induced alterations in urethra-innervating neuronal pathways should be further clarified in future studies.

\section{CLINICAL STUDY}

Diabetic neuropathy has been reported in approximately $8 \%$ patients at the diagnosis of DM. However, electrophysiologic evidence of neuropathy can be demonstrated in most patients after 5-10 years of DM [60]. Although abnormal function of bladder afferent pathways is viewed as a key clinical manifestation in DBD [21]. Moreover, positive afferent feedback from the urethra may also be compromised by diabetic neuropathy in DM rats [61]. However, the clinical evidences related to abnormal function of urethral afferent pathways in DM have not been established.

Abundant evidences showed the abnormal function of urethra efferent pathways is related to diabetic neuropathy. An earlier investigation including a series of 30 male diabetics found that fifty percent of the patients had large areflexic bladders, and increased duration of the motor unit potentials (MUP) in the periurethral striated sphincter, the striated anal sphincter, and the levator ani [62]. Diabetic vesicourethral dysfunction is highly correlated with decreased motor nerve conduction velocity in the tibial nerves, which is indicative of DM induced somatic neuropathy [63]. Diabetic polyneuropathy affects the pudendal nerve, as detected by external anal sphincter-EMG recordings, which showed an increase in MUP mean duration, mean amplitude, mean phases, satellite rate, and in percentage of long duration MUPs and polyphasic potentials [64].

In addition, DM has traditionally been handled as a peripheral metabolic disease. However, more recently, noninvasive brain imaging techniques providing information on brain anatomy and function have indicated structural and functional abnormalities associated with DM. Functional MRI studies have also shown that the hypothalamus is more sensitive to glucose concentration changes in patients with type 1 diabetes than in nondiabetic controls [65]. Since hypothalamus is closely related to micturition, modulation of central autonomic circuitry including the hypothalamus represents a potential therapeutic target for managing glucose metabolism and LUT function in diabetic patients although further studies are needed to explore CNS pathophysiology in DM-induced LUT dysfunction.

\section{CONCLUSIONS}

DM can induce the time-dependent impairment of urethral function, which can significantly contribute to DM-induced LUT dysfunction and symptoms. The underlying mechanisms are multifactorial, including time-dependent functional and morphological changes in the urethra, and the alterations of neurotransmitter systems and urethra-to-bladder reflexes. However, compared with the previous studies on DM-induced bladder dysfunction, the research on DUD is much limited. Further understandings of the molecular and functional mechanisms of DUD will be mandated to identify potential targets for therapeutic intervention of DM-related LUT dysfunction including DUD.

\section{REFERENCES}

1. Franc C. Epidemiology of diabetes: frightening predictions. Med Sci (Paris) 2013;29:711-4.

2. Frimodt-Møller C. Diabetic cystopathy: epidemiology and related disorders. Ann Intern Med 1980;92(2 Pt 2):318-21.

3. Ueda T, Yoshimura N, Yoshida O. Diabetic cystopathy: relationship to autonomic neuropathy detected by sympathetic skin response. J Urol 1997;157:580-4.

4. Daneshgari F, Liu G, Imrey PB. Time dependent changes in diabetic cystopathy in rats include compensated and decompensated bladder function. J Urol 2006;176:380-6. 
5. Liu G, Daneshgari F. Temporal diabetes- and diuresis-induced remodeling of the urinary bladder in the rat. Am J Physiol Regul Integr Comp Physiol 2006;291:R837-43.

6. Christ GJ, Hsieh Y, Zhao W, Schenk G, Venkateswarlu K, Wang $\mathrm{HZ}$, et al. Effects of streptozotocin-induced diabetes on bladder and erectile (dys)function in the same rat in vivo. BJU Int 2006;97: 1076-82.

7. Daneshgari F, Huang X, Liu G, Bena J, Saffore L, Powell CT. Temporal differences in bladder dysfunction caused by diabetes, diuresis, and treated diabetes in mice. Am J Physiol Regul Integr Comp Physiol 2006;290:R1728-35.

8. Torimoto K, Fraser MO, Hirao Y, De Groat WC, Chancellor MB, Yoshimura N. Urethral dysfunction in diabetic rats. J Urol 2004; 171:1959-64.

9. Torimoto K, Hirao Y, Matsuyoshi H, de Groat WC, Chancellor $\mathrm{MB}$, Yoshimura N. alpha1-Adrenergic mechanism in diabetic urethral dysfunction in rats. J Urol 2005;173:1027-32.

10. International Diabetes Federation. IDF diabetes atlas. 7th ed. Brussels (Belgium): International Diabetes Federation; 2015.

11. Andersen JT, Bradley WE. The syndrome of detrusor-sphincter dyssynergia. J Urol 1976;116:493-5.

12. Yang Z, Dolber PC, Fraser MO. Diabetic urethropathy compounds the effects of diabetic cystopathy. J Urol 2007;178:2213-9.

13. Gu D, Reynolds K, Duan X, Xin X, Chen J, Wu X, et al. Prevalence of diabetes and impaired fasting glucose in the Chinese adult population: International Collaborative Study of Cardiovascular Disease in Asia (InterASIA). Diabetologia 2003;46:1190-8.

14. Yang W, Lu J, Weng J, Jia W, Ji L, Xiao J, et al. Prevalence of diabetes among men and women in China. N Engl J Med 2010;362:1090101.

15. Guariguata L, Whiting DR, Hambleton I, Beagley J, Linnenkamp U, Shaw JE. Global estimates of diabetes prevalence for 2013 and projections for 2035. Diabetes Res Clin Pract 2014;103:137-49.

16. Lifford KL, Curhan GC, Hu FB, Barbieri RL, Grodstein F. Type 2 diabetes mellitus and risk of developing urinary incontinence. J Am Geriatr Soc 2005;53:1851-7.

17. Hill SR, Fayyad AM, Jones GR. Diabetes mellitus and female lower urinary tract symptoms: a review. Neurourol Urodyn 2008;27:3627.

18. Yang Z, Dolber PC, Fraser MO. Differential vulnerabilities of urethral afferents in diabetes and discovery of a novel urethra-to-urethra reflex. Am J Physiol Renal Physiol 2010;298:F118-24.

19. Daneshgari F, Moore C. Diabetic uropathy. Semin Nephrol 2006; 26:182-5.

20. Liu G, Daneshgari F. Alterations in neurogenically mediated con- tractile responses of urinary bladder in rats with diabetes. Am J Physiol Renal Physiol 2005;288:F1220-6.

21. Hanna-Mitchell AT, Ruiz GW, Daneshgari F, Liu G, Apodaca G, Birder LA. Impact of diabetes mellitus on bladder uroepithelial cells. Am J Physiol Regul Integr Comp Physiol 2013;304:R84-93.

22. Liu G, Lin YH, Yamada Y, Daneshgari F. External urethral sphincter activity in diabetic rats. Neurourol Urodyn 2008;27:429-34.

23. Latifpour J, Nishimoto T, Marian MJ, Yoshida M, Weiss RM. Differential regulation of bladder beta-adrenergic and muscarinic cholinergic receptors in experimental diabetes. Diabetes 1991;40: 1150-6.

24. Brading AF. The physiology of the mammalian urinary outflow tract. Exp Physiol 1999;84:215-21.

25. Brading AF, Teramoto N, Dass N, McCoy R. Morphological and physiological characteristics of urethral circular and longitudinal smooth muscle. Scand J Urol Nephrol Suppl 2001;(207):12-8.

26. Mamas MA, Reynard JM, Brading AF. Nitric oxide and the lower urinary tract: current concepts, future prospects. Urology 2003;61: 1079-85.

27. Abdel-Hakim A, Hassouna M, Rioux F, St-Pierre S, Abdel-Rahman $\mathrm{M}$, Galeano C, et al. Response of urethral smooth muscles to pharmacological agents. II. Noncholinergic, nonadrenergic agonists and antagonists. J Urol 1983;130:988-91.

28. Persson CG, Andersson KE. Adrenoceptor and cholinoceptor mediated effects in the isolated urethra of cat and guinea-pig. Clin Exp Pharmacol Physiol 1976;3:415-26.

29. Bennett BC, Kruse MN, Roppolo JR, Flood HD, Fraser M, de Groat WC. Neural control of urethral outlet activity in vivo: role of nitric oxide. J Urol 1995;153:2004-9.

30. Pegorare AB, Gonçalves MA, Suaid CA, Rodrigues Junior AA, Tucci S Jr, Suaid HJ, et al. Urethral dysfunction due to alloxan-induced diabetes. Urodynamic and morphological evaluation. Acta Cir Bras 2014;29:457-64.

31. Rodrigues AA Jr, Suaid HJ, Fazan VP, Reis RB, Cologna AJ, Tucci S Jr, et al. Histologic study of urethral extracellular matrix and collagen from aging and long-term alloxan-induced diabetic male rats. Urology 2011;77:510.e6-11.

32. Marini G, Piculo F, Vesentini G, Barbosa AM, Damasceno DC, Matheus SM, et al. Effects of short-term severe and long-term mild STZ-induced diabetes in urethral tissue of female rats. Neurourol Urodyn 2017;36:574-9.

33. Bortolini MA, Bilhar AP, Castro RA. Neural control of lower urinary tract and targets for pharmacological therapy. Int Urogynecol J 2014;25:1453-62.

34. de Groat WC, Griffiths D, Yoshimura N. Neural control of the low- 
er urinary tract. Compr Physiol 2015;5:327-96.

35. Sasaki K, Chancellor MB, Phelan MW, Yokoyama T, Fraser MO, Seki S, et al. Diabetic cystopathy correlates with a long-term decrease in nerve growth factor levels in the bladder and lumbosacral dorsal root Ganglia. J Urol 2002;168:1259-64.

36. Förstermann U, Closs EI, Pollock JS, Nakane M, Schwarz P, Gath I, et al. Nitric oxide synthase isozymes. Characterization, purification, molecular cloning, and functions. Hypertension 1994;23(6 Pt 2):1121-31

37. Förstermann U, Sessa WC. Nitric oxide synthases: regulation and function. Eur Heart J 2012;33:829-37, 837a-837d.

38. Persson K, Alm P, Uvelius B, Andersson KE. Nitrergic and cholinergic innervation of the rat lower urinary tract after pelvic ganglionectomy. Am J Physiol 1998;274:R389-97.

39. Gillespie JS, Liu XR, Martin W. The effects of L-arginine and NGmonomethyl L-arginine on the response of the rat anococcygeus muscle to NANC nerve stimulation. Br J Pharmacol 1989;98:10802.

40. Warner TD, Mitchell JA, Sheng H, Murad F. Effects of cyclic GMP on smooth muscle relaxation. Adv Pharmacol 1994;26:171-94.

41. Zhang B, Zhang Z, Ji H, Shi H, Chen S, Yan D, et al. Grape seed proanthocyanidin extract alleviates urethral dysfunction in diabetic rats through modulating the NO-cGMP pathway. Exp Ther Med 2018;15:1053-61.

42. Igawa Y, Yamazaki Y, Takeda H, Hayakawa K, Akahane M, Ajisawa $\mathrm{Y}$, et al. Functional and molecular biological evidence for a possible beta3-adrenoceptor in the human detrusor muscle. Br J Pharmacol 1999;126:819-25.

43. Testa R, Guarneri L, Ibba M, Strada G, Poggesi E, Taddei C, et al. Characterization of alpha 1-adrenoceptor subtypes in prostate and prostatic urethra of rat, rabbit, dog and man. Eur J Pharmacol 1993;249:307-15.

44. Yashiro K, Thor KB, Burgard EC. Properties of urethral rhabdosphincter motoneurons and their regulation by noradrenaline. J Physiol 2010;588(Pt 24):4951-67.

45. Chen S, Zhu Y, Feng X, Zhang Z, Li S, Shi B. Changes in alpha1adrenoceptor and NGF/proNGF pathway: a possible mechanism in diabetic urethral dysfunction. Urol Int 2014;93:344-51.

46. Schäper C, Gläser S, Groneberg DA, Kunkel G, Ewert R, Noga O. Nerve growth factor synthesis in human vascular smooth muscle cells and its regulation by dexamethasone. Regul Pept 2009;157:37.

47. Matsuyama A, Takatori S, Sone Y, Ochi E, Goda M, Zamami Y, et al. Effect of nerve growth factor on innervation of perivascular nerves in neovasculatures of mouse cornea. Biol Pharm Bull 2017;
40:396-401.

48. Li Y, Shi B, Wang D, Wang P, Laudon V, Zhang J, et al. Nerve growth factor and substance P: expression in a rat model of diabetic bladder. Int Urol Nephrol 2011;43:109-16.

49. Quarta S, Baeumer BE, Scherbakov N, Andratsch M, Rose-John S, Dechant G, et al. Peripheral nerve regeneration and NGF-dependent neurite outgrowth of adult sensory neurons converge on STAT3 phosphorylation downstream of neuropoietic cytokine receptor gp130. J Neurosci 2014;34:13222-33.

50. Steers WD, Tuttle JB. Mechanisms of Disease: the role of nerve growth factor in the pathophysiology of bladder disorders. Nat Clin Pract Urol 2006;3:101-10.

51. Brading AF. Spontaneous activity of lower urinary tract smooth muscles: correlation between ion channels and tissue function. J Physiol 2006;570(Pt 1):13-22.

52. Hellweg R, Hartung HD. Endogenous levels of nerve growth factor (NGF) are altered in experimental diabetes mellitus: a possible role for NGF in the pathogenesis of diabetic neuropathy. J Neurosci Res 1990;26:258-67.

53. Hellweg R, Raivich G, Hartung HD, Hock C, Kreutzberg GW. Axonal transport of endogenous nerve growth factor (NGF) and NGF receptor in experimental diabetic neuropathy. Exp Neurol 1994;130:24-30.

54. Tong YC, Cheng JT. Changes in bladder nerve-growth factor and p75 genetic expression in streptozotocin-induced diabetic rats. BJU Int 2005;96:1392-6.

55. Nirmal J, Tyagi P, Chuang YC, Lee WC, Yoshimura N, Huang CC, et al. Functional and molecular characterization of hyposensitive underactive bladder tissue and urine in streptozotocin-induced diabetic rat. PLoS One 2014;9:e102644.

56. Bruns TM, Bhadra N, Gustafson KJ. Bursting stimulation of proximal urethral afferents improves bladder pressures and voiding. J Neural Eng 2009;6:066006.

57. Shafik A, Shafik AA, El-Sibai O, Ahmed I. Role of positive urethrovesical feedback in vesical evacuation. The concept of a second micturition reflex: the urethrovesical reflex. World J Urol 2003;21: 167-70.

58. Pinna C, Bolego C, Puglisi L. Effect of substance P and capsaicin on urinary bladder of diabetic rats and the role of the epithelium. Eur J Pharmacol 1994;271:151-8.

59. Al-Noah Z, McKenna D, Langdale C, Thor KB, Marson L, Burgard E, et al. Nitrergic relaxations and phenylephrine contractions are not compromised in isolated urethra in a rat model of diabetes. Auton Neurosci 2014;183:58-65.

60. Factors in development of diabetic neuropathy. Baseline analysis of 
neuropathy in feasibility phase of Diabetes Control and Complications Trial (DCCT). The DCCT Research Group. Diabetes 1988; 37:476-81.

61. Jung SY, Fraser MO, Ozawa H, Yokoyama O, Yoshiyama M, De Groat WC, et al. Urethral afferent nerve activity affects the micturition reflex; implication for the relationship between stress incontinence and detrusor instability. J Urol 1999;162:204-12.

62. Fagerberg SE, Kock NG, Peteson I, Stener I. Urinary bladder disturbances in diabetics: I. A comparative study of male diabetics and controls aged between 20 and 50 years. Scand J Urol Nephrol 1967; 1:19-27.
63. Mitsui T, Kakizaki H, Kobayashi S, Morita H, Matsumura K, Koyanagi T. Vesicourethral function in diabetic patients: association of abnormal nerve conduction velocity with vesicourethral dysfunction. Neurourol Urodyn 1999;18:639-45.

64. Jian F, Pan H, Zhang Z, Lin J, Chen N, Zhang L, et al. Sphincter electromyography in diabetes mellitus and multiple system atrophy. Neurourol Urodyn 2015;34:669-74.

65. Musen G, Simonson DC, Bolo NR, Driscoll A, Weinger K, Raji A, et al. Regional brain activation during hypoglycemia in type 1 diabetes. J Clin Endocrinol Metab 2008;93:1450-7. 